\title{
The Future of Laboratory Information Systems: User Input Impacting the Development of Laboratory Information Systems
}

\author{
Megan Patterson \\ Cirdan \\ Lisburn, N. Ireland \\ mpatterson@cirdan.com
}

\begin{abstract}
The development of professional medical software is often behind the curve in both technical and design implementation. This is in part due to the slow rate of replacement of software within medical institutions. Additionally, users are often not consulted in the development of these types of software. In the case of medical software, this can often have unforeseen and unfortunate consequences. User Experience research shows that involving users at every stage in the development of software leads to improved effectiveness, improved overall usability, and compliance with regulatory standards. By carrying out workshops and surveys, Cirdan has investigated which features have the highest priority for users within the development of Laboratory Information Systems (LIS). The results of this research are having a direct influence on the future of LIS within Cirdan.
\end{abstract}

Laboratory Information System, User Experience, User Experience design, Usability, pathology

\section{INTRODUCTION}

The development of complex medical software can often be extremely challenging. There are often many considerations for both technical and interface designs. This complexity often means that these types of software are often "behind the curve" when it comes to modern design techniques. In addition to this, the users of this software are often not consulted when new developments are being made. This lack of user input can have disastrous consequences, leading to software which is not fit for purpose, or is difficult to operate without extensive specialist training. This paper examines the efforts of one company, Cirdan, as they attempt to improve the development of their Laboratory Information System (LIS) by incorporating key User Experience (UX) techniques into their development processes to include users and establish the overall future of LIS.

\section{BACKGROUND}

A LIS is a complex software program which is used to record, manage, and store data within a clinical laboratory, typically being used in pathology and its various sub-disciplines. A LIS will often need to be specifically configured to meet the needs of individual laboratories. These computerized systems have been introduced in laboratories as a way of managing and tracking specimens as they move through the laboratory process. They also somewhat reduce the amount of paper records required in the laboratory.

The first attempts at the creation of LIS technology was in the 1960s. However, most of them were adapted from larger Hospital Information Systems and had no functionality specific to clinical laboratories. Park et al. (2012) stated that the two main reasons for a lack of success at LIS development were (1) a lack of technical understanding in the implementation of the system (2) "the lack of communication between the providers and the end users". Clearly, the issue surrounding customer involvement in software development is a long-standing one.

Many interfaces for LIS began as character-based interfaces. These type of interfaces were controlled entirely using a keyboard. With experience and training, this type of control proved to be highly efficient. With advancements in technology, many LIS stared upgrading to a Graphical User Interface (GUI). This allowed for more advanced display 
capabilities as well as the ability to navigate the software using a mouse. Many interfaces which were initially given a GUI in the 1990s and early 2000s have not been redesigned since, leading to dated and difficult to use interfaces. With recent technological improvements, many companies are not looking to moving their LIS to the web.

The adoption of new technology is often slow in the healthcare sector. Due to the complex nature of hospital systems, integrating new technologies can be a complex and time-consuming process. there are also patient-centric considerations to take into account around safety and security which can discourage implementing new systems (Thurston \& Gagnon, 2017).

\section{IMPORTANCE OF USER EXPERIENCE IN DEVELOPMENT}

User Experience is becoming increasingly important in software development, particularly in regards to developing software for the healthcare industry. Interfaces which are difficult to use, interpret, or understand can not only lead to users having a poor experience, but can also compromise user safety.

There are different methods for bringing UX into development. One of these is the idea of participatory design. This is "a set of theories, practices, and studies related to end-users as full participants in activities leading to software and hardware computer products" (Muller \& Kuhn, 1993). By having those users who will ultimately be using the end product have a direct input into its creation, they will end up with a product that fulfils their needs. Studies into development projects which have incorporated participatory design have reported success; citing their ability to manage challenge more effectively (Gennari \& Reddy, 2000). One study raised the issue of "change management" as a major factor in the use of participatory design in healthcare particularly, stating "any system's installation may affect a very big pool of users with diverse training backgrounds, different levels of expertise, and varied system requirements" (Weng et al., 2007).

Table 1: Factors broken down into categories

\section{THE FUTURE OF LIS}

Clearly, there are many challenges facing the future of LIS, both in terms of current UX failings and future LIS development. To help identify some of the key concerns coming from those using the systems in their daily work, Cirdan conducted a study on "The Future of LIS". This was carried out in two stages; the first was a workshop which allowed Cirdan employees to openly discuss with health practitioners the current issues with many LIS systems and what key features they would require from their LIS in the future. The second stage involved the construction of a survey based on the data collected from the workshop.

\subsection{Workshop}

The workshop was carried out as part of the Diagnostics Boot Camp 2017 event (Ukas.com, 2017). This workshop allowed participants to discuss the future of LIS centring around five core stages of the laboratory process (1) Referrals: this deals with the ordering of tests and various related information. (2) Pre-analytical: includes items which are required before specimens are tested (3) Analytical: factors which need to be considered during the testing of specimens (4) Post-Analytical: factors which are required once testing is completed (5) Other: additional factors which have an impact on the laboratory and the testing process.

\subsection{Survey}

After the workshop, a list of factors was collated under the five headings. These factors can be viewed in Table 1. it was decided to expand the discussion and validate the workshop findings by circulating the factors to the wider pathology community. A survey was developed using the core stages and their factors. Survey-takers were asked to rate each factor as very important, important, somewhat important, or not important. Each of these rating were weighted, with not important being 1 , and very important being 4 . Additionally, the survey provided the opportunity for participants to mention any additional factors they felt were of importance to the future of LIS that had not been included in the survey.

\begin{tabular}{|l|l|l|l|l|}
\hline Referrals & Pre-Analytical & Analytical & Post-Analytical & Other \\
\hline eOrder Comms & $\begin{array}{l}\text { Specimen Collection } \\
\text { App }\end{array}$ & POCT Assays & eResults Reporting & Security \\
\hline $\begin{array}{l}\text { Access To Test } \\
\text { Guides }\end{array}$ & Courier Management & $\begin{array}{l}\text { Deep Learning } \\
\text { Module }\end{array}$ & Billing & $\begin{array}{l}\text { Data Mining } \\
\text { Capability }\end{array}$ \\
\hline Status Updates & Flexible Accessioning & $\begin{array}{l}\text { Integrated Method } \\
\text { Validation }\end{array}$ & Registry Submissions & Good Interoperability \\
\hline $\begin{array}{l}\text { Appointment } \\
\text { Management }\end{array}$ & Specimen Routing & $\begin{array}{l}\text { Comprehensive QC } \\
\text { Techniques }\end{array}$ & Notifications & Cost Of Ownership \\
\hline Paper Requests & RFID Management & Multi-lab Solution & Patient Access & Highly Configurable \\
\hline Quotation & Snomed CT Encoder & Manual Results Entry & $\begin{array}{l}\text { Resource } \\
\text { Management }\end{array}$ & Ready Installed \\
\hline & & & & Cloud \\
\hline
\end{tabular}




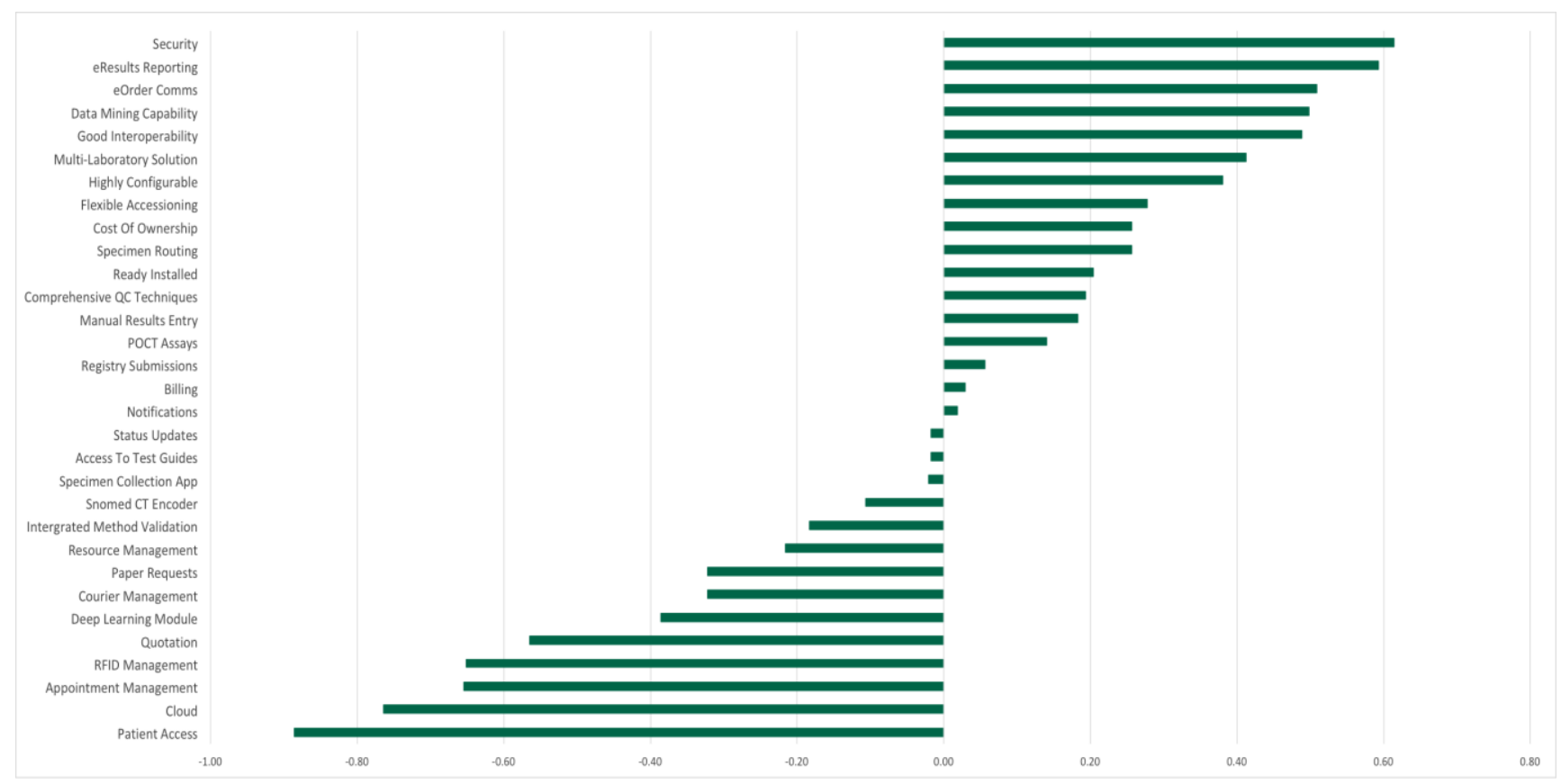

Figure 1: Weighted averages of factors considered in Future of LIS survey

\section{RESULTS}

In total, 95 participants completed the survey. The ratings were analysed to calculate the average score for each factor. From this, a weighted average was calculated. These resulted in a graph which depicts the factors of high significance and those of low significance (Figure 1). The five most important and two least important factors were examined in more detail.

\subsection{Security}

Having a LIS with high security measures was rated the most important factor by survey participants. Sametinger et al. (2015) discuss the importance of good security within medical software. They highlight that this is not only about the protection of "sensitive health information", but is also about "protecting human life". In user experience terms, it is important to balance the level of security required by a LIS with the systems overall usability. It is crucial that security levels do not impede users from carrying out their work, or compromise the speed at which pathology laboratories often need to operate.

\section{2 eOrders \& eResults Communications}

The ability to digitally order laboratory tests and receive the results digitally have become highly important in the laboratory workflow. This is particularly true with the introduction of Electronic Health Records (EHR), which centralise patient information, as it allows for the entire workflow to move from the traditional paper-based systems. This change, of course, has its own challenges. In a study examining the removal of paper-based systems in two Norwegian hospitals showed that, while the introduction of digital systems was a success, "many of the old routines remain unchanged" (Lium, Tjora, \& Faxvagg, 2008). This limited the overall effectiveness of the digital solutions. It should, therefore, be noted that if the laboratory process is to be completely digitised, it will require a cultural change in addition to a technological one.

\subsection{Data Mining Capability}

Data mining is the technique of identifying patterns and understanding relationships present in data by conducting effective data analysis techniques. This is an aspect which survey participants found highly important, as effect data mining of medical data has the potential to help aid diagnosis and increase medical knowledge (Prather et al., 1997). However, there are challenges with effectively mining medical data as it can be widely distributed across many medical networks, and is often diverse in nature and of a very high volume (Wasan, Bhatnagar, \& Kaur, 2006). There are also issues around the medical data mining from an ethical standpoint due to the deeply personal nature of the data. Therefore, in order to provide an effective data mining capability, the LIS must also have effective security in place. 


\subsection{Good Interoperability}

Interoperability is becoming a major issue as many systems now require communication with each other to share important data. In the development of their medical cyber-physical systems, Lee et al. (2012) outlined the importance of good interoperability and how they developed an "interoperability manager" to coordinate all the systems on the network. Building a similar manager into a LIS would provide similar benefits by coordinating medical software, hospital computer systems, and medical instruments. Good interoperability also aids in providing a positive user experience as all these components of the laboratory work together seamlessly, with little to no need for user intervention.

\subsection{Highly Configurable}

Each laboratory has its own unique laboratory process and workflow. It is, therefore, vital that key software programs can be configured to facilitate individual laboratories and their way of working. This is the category that falls most neatly into the traditional area of usability and user experience. Working closely with users to determine their exact needs and attempting to facilitate them using the LIS is a complex and potentially time-consuming exercise. As with the previous categoires, a balance needs to made between user requirements and business requirements. By employing key UX research techniques, a "happy path" for different workflows in different laboratories could be established and built upon to try and consolidate similarities between laboratories.

\subsection{Patient Access}

With the increase in health technologies in recent years, the concept of e-health is becoming an increasingly important one. Now more than ever, patients are taking a real interest in their health and the data surrounding it. This has led to an increasing desire in patients to have access to all their health records, including the medical tests being carried out on them. Providing patient access to a LIS was rated of least importance overall by survey participants. However, various legislations are now making it mandatory for patients to be allowed access to their own information. Studies carried out to ascertain the benefits of providing patient access showed an increase in the quality of communication between doctors and patients, as well as providing an overall positive user experience (Ross \& Lin, 2003). In UX terms, it is important to note that giving patient access to a complex system such as a LIS would provide a different set of challenges due to their lack of detailed medical knowledge.

\subsection{Cloud}

Cloud computing is becoming increasingly prominent in computing today as it allows information to be held and accessed from an external source, as well as providing the ability to access data from wider geographic locations. These advantages help to provide a positive user experience as they provide users with a more flexible way of working. Despite these benefits, survey participants rated this as one of the least important factors. This may be due, in part, to the potential security issues a cloud solution may present. Research carried out by Almorsy, Grundy, and Müller (2016) discussed some of the key security concerns with the cloud and suggested some potential methods of rectifying these issues. It is clear, therefore, that if a LIS with good security measures was developed, that a cloud-based solution to data storage may become a more desirable factor.

\section{CONCLUSIONS AND FUTURE WORK}

This paper has attempted to describe the challenges faced in the development of medical software. It focused on the development of LIS and how the introduction of user input could have a positive impact on the future of LIS development. By running the workshop, Cirdan was able to have a direct discussion with users to establish a list of concerns within key areas of the laboratory workflow. In opening the debate up to the wider community, this list of concerns was validated and prioritised. The five main concerns have been investigated in more detail, and will now be taken forward by Cirdan into their development process. Continuing to include users as these factors are developed is key for Cirdan to develop an effective product and have a positive influence on the future of LIS.

\section{REFERENCES}

Almorsy, M., Grundy, J. and Müller, I., 2016. An analysis of the cloud computing security problem. arXiv preprint arXiv:1609.01107.

Gennari, J.H. and Reddy, M., 2000. Participatory design and an eligibility screening tool. In Proceedings of the AMIA symposium (p. 290). American Medical Informatics Association.

Lee, I., Sokolsky, O., Chen, S., Hatcliff, J., Jee, E., Kim, B., King, A., Mullen-Fortino, M., Park, S., Roederer, A. and Venkatasubramanian, K.K., 2012. Challenges and research directions in medical cyber-physical systems. Proceedings of the IEEE, 100(1), pp.75-90. 
Muller, M.J. and Kuhn, S., 1993. Participatory design. Communications of the ACM, 36(6), pp.24-28.

Park, S.L., Pantanowitz, L., Sharma, G. and Parwani, A.V., 2012. Anatomic pathology laboratory information systems: a review. Advances in anatomic pathology, 19(2), pp.81-96.

Prather, J.C., Lobach, D.F., Goodwin, L.K., Hales, J.W., Hage, M.L. and Hammond, W.E., 1997. Medical data mining: knowledge discovery in a clinical data warehouse. In Proceedings of the AMIA annual fall symposium (p. 101). American Medical Informatics Association.

Ross, S.E. and Lin, C.T., 2003. The effects of promoting patient access to medical records: a review. Journal of the American Medical Informatics Association, 10(2), pp.129-138.

Sametinger, J., Rozenblit, J., Lysecky, R. and Ott, P., 2015. Security challenges for medical devices. Communications of the ACM, 58(4), pp.74-82.

Thurston, G. and Gagnon, H. (2017). From Behind The Curve To Beyond The Pill. [online] Popewoodhead.com. Available at: https://www.popewoodhead.com/files/Digital_Pat ient-centered_models.pdf [Accessed 27 Feb. 2018].

Ukas.com. (2017). UKAS : Diagnostics Boot Camp 2017: 22nd November 2017, London. [online] Available at: https://www.ukas.com/news/133862/ [Accessed 21 Mar. 2018].

Wasan, S.K., Bhatnagar, V. and Kaur, H., 2006. The impact of data mining techniques on medical diagnostics. Data Science Journal, 5, pp.119-126.

Weng, C., McDonald, D.W., Sparks, D., McCoy, J. and Gennari, J.H., 2007. Participatory design of a collaborative clinical trial protocol writing system. International Journal of Medical Informatics, 76, pp.S245-S251. 